\title{
Quantitative shear wave elastography in primary invasive breast cancers, based on collagen-S100A4 pathology, indicates axillary lymph node metastasis
}

\author{
Xin Wen ${ }^{1,2}$, Xiwen Yu ${ }^{3}$, Yuhang Tian ${ }^{2}$, Zhao Liu ${ }^{2}$, Wen Cheng ${ }^{2}$, Hairu Li ${ }^{1}$, Jia Kang ${ }^{1}$, Tianci Wei ${ }^{1}$, \\ Shasha Yuan', Jiawei Tian ${ }^{1}$ \\ ${ }^{1}$ Department of Ultrasound, The Second Affiliated Hospital of Harbin Medical University, Harbin 150001, China; ${ }^{2}$ Department of Ultrasound, \\ Harbin Medical University Cancer Hospital, Harbin 150081, China; ${ }^{3}$ Heilongjiang Academy of Medical Sciences, Harbin 150086, China
}

Correspondence to: Jiawei Tian. Department of Ultrasound, The Second Affiliated Hospital of Harbin Medical University, 148 Baojian Rd, Nangang District, Harbin 150001, China. Email: jwtian2004@163.com.

Background: The purpose of this study was to evaluate the value of quantitative shear wave elastography (SWE) in indicating the axillary lymph node metastasis (LNM) of invasive breast cancers (IBCs) and to investigate if S100A4 plays a key role in promoting metastasis and increasing stiffness in IBC.

Methods: The differences in SWE of 223 IBC patients were compared between the LNM+ and LNMgroups and the optimal cutoff values of SWE for diagnosing LNM were calculated. We searched the gene expression omnibus (GEO) to determine whether S100A4 was more highly expressed in IBCs that were $\mathrm{LNM}+$ than in those that were LNM-. Sirius red and immunohistochemical staining were used to examine the collagen deposition and S100A4 expression of included tissue samples, and correlations of SWE and S100A4 expression with collagen deposition were analyzed.

Results: The optimal cutoff values for Emax (the maximum stiff value), Emean (the mean stiff value), and EmeanR (the ratio of Emean between mass and parenchyma) for diagnosing axillary LNM were $111.05 \mathrm{kPa}$, $79.80 \mathrm{kPa}$, and 6.89, respectively. GSE9893 exhibited more increased S100A4 expression in IBCs that were $\mathrm{LNM}+$ than in those that were LNM-. Collagen volume fraction (CVF) and the average optical density of S100A4 $\left(\mathrm{AOD}_{\left.\mathrm{S}_{100 \mathrm{~A}}\right)}\right)$ in the LNM+ group were significantly higher than those in the LNM- group. Emax, Emean, EmeanR, and $\mathrm{AOD}_{\mathrm{S} 100 \mathrm{~A}^{4}}$ were all positively correlated with CVF.

Conclusions: SWE in primary IBC could be useful for indicating axillary LNM. S100A4 may be a factor that regulates cancer-associated collagen deposition and metastasis; however, prospective molecular biological studies are needed.

Keywords: Invasive breast cancer (IBC); lymph node metastasis (LNM); shear wave elastography (SWE); S100A4; collagen

Submitted Jul 17, 2019. Accepted for publication Feb 18, 2020.

doi: 10.21037 /qims.2020.02.18

View this article at: http://dx.doi.org/10.21037/qims.2020.02.18

\section{Introduction}

The accurate preoperative identification of invasive breast cancers (IBCs) with lymph node metastasis (LNM) could not only minimize the need for clearing axilla after breast surgery but also influence the use of systemic treatment, such as neoadjuvant therapy. Among several imaging methods, ultrasound (US) is more routinely used to diagnose LNM preoperatively. Shear wave elastography (SWE), which uses acoustic radiation force induced by US beams, induces shear waves to propagate transversely in the tissue and has been commonly used to differentiate between benign and malignant tumors (1). It is thought that 


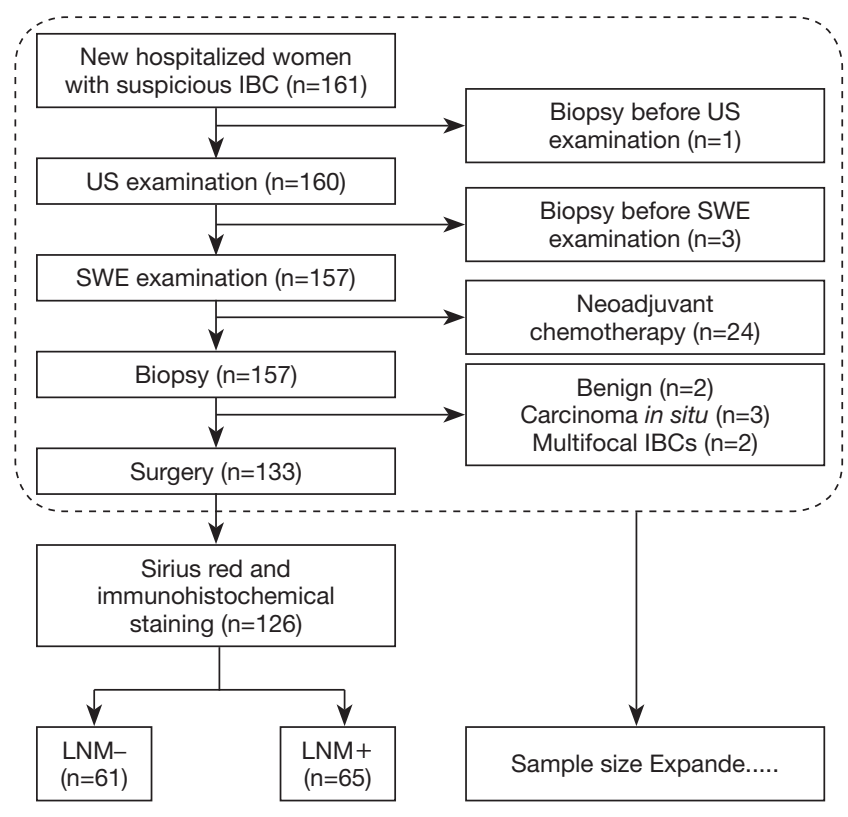

Figure 1 The study flow chart. IBC, invasive breast cancer; US, ultrasound; SWE, shear wave elastography; LNM, lymph node metastasis.

the stromal reaction that occurs due to invasive behavior results in abnormal tumor-associated collagen deposition, potentially leading to increased SWE values in IBC (2). Studies have shown that Emean, one elasticity index of SWE, could be useful for predicting the nodal status of IBCs, but the optimal cutoff value to diagnose LNM along with the pathological basis and possible molecular mechanism for lymphatic metastasis prediction, are still unknown (3).

S100A4 is a member of the $\mathrm{S} 100$ protein family, which is well known for its significant role in promoting cancer progression and metastasis (4). Many studies have shown that S100A4 plays an important role in epithelialmesenchymal transition (EMT), which is associated with tissue fibrosis by stimulating the deposition of collagen in nontumor pathophysiologies, such as rheumatoid arthritis and systemic sclerosis $(5,6)$. However, whether S100A4 plays a regulatory role in collagen deposition in tumors is unknown. Therefore, we hypothesized that S100A4 simultaneously promotes metastasis and hardens primary IBCs by remodeling cancer-associated collagen deposition.

To test this, our study aimed to both determine the optimal cutoff values of SWE for diagnosing LNM and to investigate the pathological basis and key molecules involved.

\section{Methods}

We prospectively observed 126 hospitalized women with a postoperative pathological diagnosis of IBC in the Harbin Medical University Cancer Hospital. Specialist ultrasonographers examined preoperative USs in our ultrasound department, and then the subjects were scanned in another room using SWE by the same doctor (with 4 years of SWE experience). A dedicated pathologist oversaw immunohistochemical staining of all included pathological slides. The doctors were blinded to each other. To determine the best cutoff values of SWE for diagnosing LNM, we further expanded the sample size to include 223 IBC patients in the cohort.

\section{Patients}

The inclusion criteria were new hospitalized women with a suspicious diagnosis of IBC who had US and SWE examinations before biopsy. The exclusion criteria included the following: biopsy before US and/or SWE, undergoing neoadjuvant chemotherapy before surgery, carcinoma in situ or benign postoperative pathological diagnosis, and patients with pathologically multifocal IBCs. The included samples were divided into LNM+ and LNM- groups depending on the presence or absence of axillary LNM as defined by postoperative pathology. The study flow chart is shown in Figure 1. In total, 126 patients with a surgical diagnosis of IBC (65 patients in the LNM+ group and 61 patients in the LNM- group) were included in the previous phase for pathology basis and possible key molecular investigation. In the later phase, 97 consecutive patients (44 patients in the LNM+ group and 53 patients in the LNM- group) were filtered into the cohort according to the inclusion and exclusion criteria, for a more reliable calculated cutoff value. Consequently, an expanded sample size with 223 patients (109 patients in the LNM+ group and 114 patients in the LNM- group) were included for SWE cutoff value determination, along with US and clinicopathological statistical analysis in this study.

All procedures performed in studies involving human participants were in accordance with the ethical standards of the institutional and/or national research committee and with the 1964 Helsinki declaration and its later amendments or comparable ethical standards. The present study was reviewed and approved by the Ethics Committee of the Harbin Medical University Cancer Hospital. Written 
informed consent was obtained from each patient.

\section{Preoperative US and SWE examination}

US and SWE examinations were conducted preoperatively using a SuperSonic Imagine ultrasound system (Aixplorer, Aixen Provence, France) equipped with an SL10-2 lineararray transducer of 2-10 MHz. Ultrasonographic features were recorded according to the 5th American College of Radiology Breast Imaging Reporting and Data System (BIRADS) lexicon (7). For the SWE examination, the display scale of the elasticity color image was set as $0-180 \mathrm{kPa}$. The region of interest (ROI) on the SWE display included the mass and a small amount of surrounding breast tissue. A Q-Box $2 \mathrm{~mm}$ in diameter was placed, and elasticity parameters, including Emax (the maximum stiff value), Emean (the mean stiff value), and EmeanR (the ratio of Emean between mass and parenchyma) were all obtained after the elastogram was stable for 3-5 seconds.

\section{Bioinformatics analysis}

To investigate whether S100A4 is more highly expressed in primary IBCs that are $\mathrm{LNM}+$ than in those that are LNM-, we searched the gene expression omnibus (GEO). First, "S100A4" was input into the GEO profiles as a keyword, with 3,741 chip results being the output. There were 42 chips correlated with tumor metastasis, only 1 of which contained data concerning the metastasis of breast carcinoma: it showed no difference between the S100A4 expression in primary lesions with metastasis and without metastasis. Then, the keywords "S100A4" and "breast cancer or carcinoma metastasis" were input into the GEO datasets, and 1,204 data points were output. GSE9893 was selected randomly to be further processed. The difference of S100A4 expression level between the primary lesions with metastasis and lesions without metastasis was calculated using 2 independent samples Student's $t$-test.

\section{Sirius red and immunobistochemical staining}

All the pathological slides included were fixed in $4 \%$ buffered paraformaldehyde, decalcified in $50 \mathrm{mM}$ ethylenediaminetetraacetic acid (EDTA), embedded in paraffin, and sectioned at $5 \mu \mathrm{m}$ thickness. Sirius red staining was used to assess collagen composition, and immunohistochemical staining was used to examine the expression of S100A4. Five high-power fields $(\times 200)$ were selected and imaged randomly on each slide. Collagen volume fraction (CVF) and average optical density of S100A4 $\left(\mathrm{AOD}_{\mathrm{S}_{100 \mathrm{~A}} 4}\right)$ in each field were quantitatively calculated using ImageJ software version 1.51 (http:// rsb.info.nih.gov/ij/). Collagen deposition and S100A4 expression are shown as average values for CVF and $\mathrm{AOD}_{\mathrm{S} 100 \mathrm{~A}_{4}}$ in 5 fields, respectively.

\section{Statistical analysis}

Correlations of collagen deposition with SWE parameters and S100A4 expression were evaluated by Pearson correlation analysis, and the coefficients were defined as follows: $<0.40$ indicated poor reliability; $0.40-0.75$ indicated good reliability, and $>0.75$ indicated excellent reliability (8). The comparisons of clinicopathological characteristics, US features, SWE values, and collagen and S100A4 expression in IBCs between the LNM+ and LNM- groups were performed using 2 independent samples Student's $t$-test or $\chi^{2}$ tests according to the presence of measurement or enumeration data. Receiver operating characteristic (ROC) curve analysis was performed to evaluate the diagnostic performance of SWE for diagnosing axillary lymphatic metastasis. All analyses in this study were performed using SPSS version 22.0 (SPSS Inc., Chicago, IL, USA) with $\mathrm{P}<0.05$ as the threshold for statistical significance.

\section{Results}

\section{S100A4 expression, collagen deposition and SWE}

GSE9893 in GEO exhibited more increased S100A4 expression in primary IBCs that were $\mathrm{LNM}+$ than in those that were LNM- $\left[\mathrm{P}=0.0008232, \log ^{2}\right.$ (fold change) $\left.=0.35\right]$. In our cohort, there were 65 tissue samples in the LNM+ group and 61 in the LNM- group consecutively included for S100A4 immunohistochemical staining and collagen staining. S100A4 expression levels were significantly higher in the LNM+ group than in the LNM- group $\left(\mathrm{AOD}_{\mathrm{S} 100 \mathrm{~A}}\right.$ : $10.15 \pm 6.64$ vs. $3.33 \pm 2.01$, respectively, $\mathrm{P}<0.001$ ). Mean collagen deposition of the 65 primary LNM+ IBC tissue samples was higher compared to that of the 61 primary LNM- IBC tissue samples (CVF: 25.65 \pm 8.83 vs. $16.39 \pm 6.08$, respectively, $\mathrm{P}<0.001)$. Collagen staining and S100A4 immunohistochemical staining of included LNM+ and LNM- samples are shown in Figure 2. The SWE parameters were positively correlated with CVF in primary IBC $(r-E m a x=0.789, \mathrm{P}<0.001 ; \mathrm{r}-\mathrm{Emean}=0.674$, 

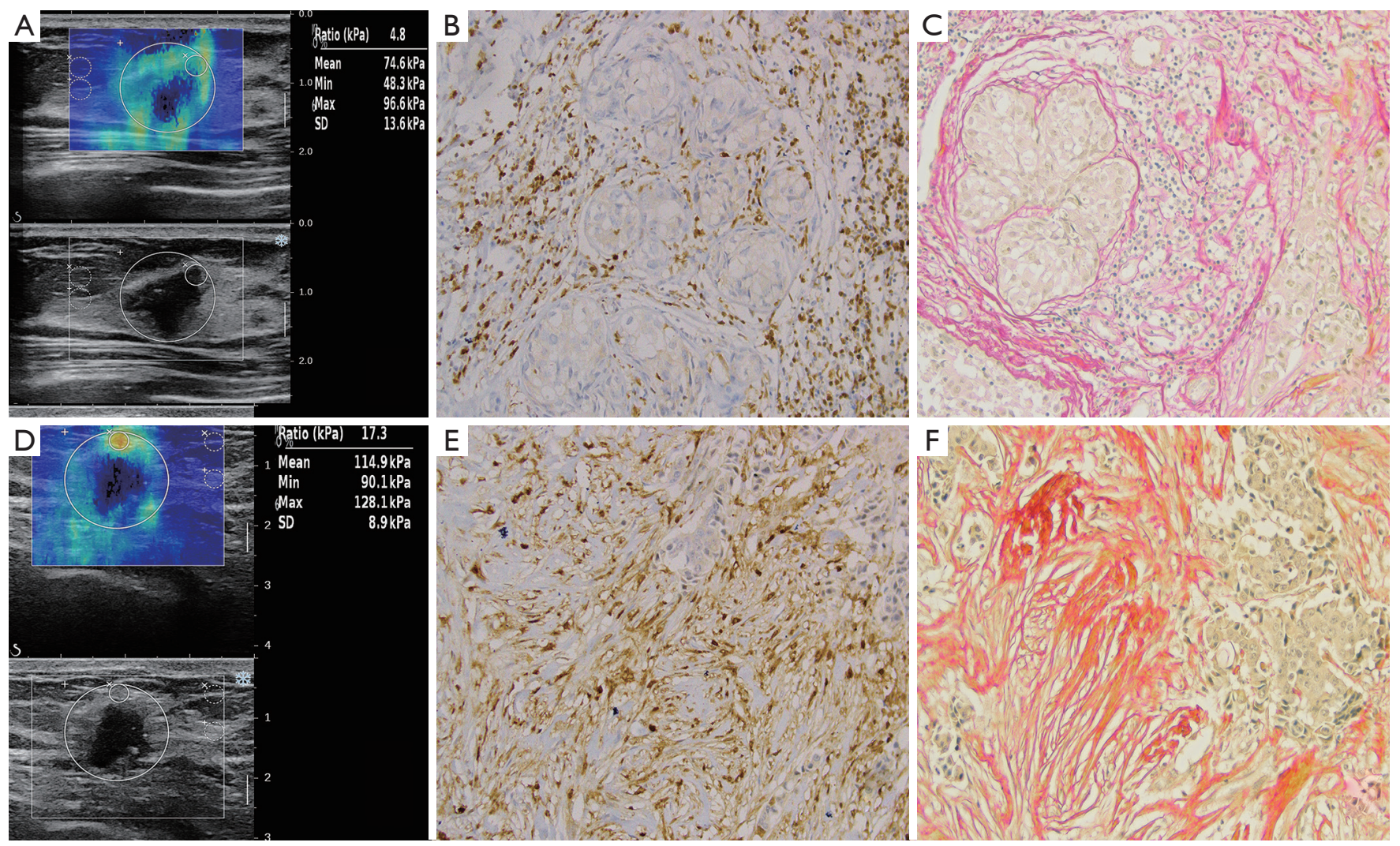

Figure 2 The comparison of SWE (A,D), S100A4 immunohistochemical staining (B,E: $\times 200)$ and collagen Sirius staining $(\mathrm{C}, \mathrm{F}$ : $\times 200)$ of IBC between the LNM- (A,B,C) and LNM+ (D,E,F) groups. SWE, shear wave elastography; IBC, invasive breast cancer; LNM, lymph node metastasis.

$\mathrm{P}<0.001 ; \mathrm{r}$-Emean $\mathrm{R}=0.443, \mathrm{P}<0.001$ ), which indicated collagen deposition was higher in stiff masses with higher SWE values. Furthermore, collagen composition was positively correlated with S100A4 expression levels ( $\mathrm{r}=0.793$, $\mathrm{P}<0.001$ ), inferring that $\mathrm{S} 100 \mathrm{~A} 4$ expressed higher in masses with more collagen composition. Correlations of elasticity indexes and CVF, along with $\mathrm{CVF}$ and $\mathrm{AOD}_{\mathrm{S100A}_{4}}$, are shown in Figure 3.

\section{Clinicopathological characteristics}

In total, 223 women pathologically diagnosed with IBC were included for analysis to determine the optimal cutoff value of SWE in this study. The median age was 55 years and ranged from 31 to 78 years. There were 109 patients (48.88\%) with axillary lymphatic metastasis in the LNM+ group and 114 patients $(51.12 \%)$ in the LNM- group. Of the 223 cases, $51.6 \%$ (115 patients, including 98 in the LNM+ group and 17 in the LNM- group) were treated with modified radical mastectomy, 36.3\% (81 patients, including 5 in the LNM+ group and 76 in the LNM- group) were treated by mastectomy with sentinel lymph node dissection, and $12.1 \%$ (27 patients, including 6 in the LNM+ group and 21 in the LNM- group) underwent breast-conserving surgery. A total of 115 (51.6\%) experienced preoperative needle biopsy on suspicious lymph nodes, 94 cases (42.1\%) were treated with intraoperative sentinel lymph node biopsy, and both of the above biopsy methods treated 14 (6.3\%, 5 in the LNM+ group and 9 in the LNM- group) cases. A comparison of clinicopathological characteristics between the LNM+ and LNM- groups is shown in Table 1. As presented, the mean tumor size in the LNM+ group was significantly larger than that in the LNM- group $(\mathrm{P}=0.018)$. Samples with lymphovascular invasion in the LNM+ group were also significantly increased compared to the LNMgroup $(\mathrm{P}<0.001)$. Furthermore, Ki-67 expression levels were increased in the LNM+ group compared to those in the LNM- group $(\mathrm{P}=0.016)$. No significant differences 


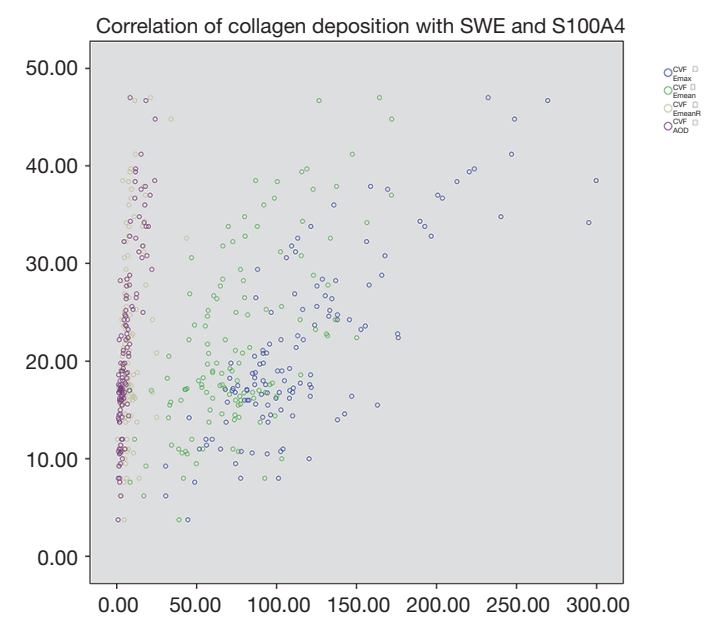

Figure 3 The correlations of Emax, Emean, EmeanR, and $\mathrm{AOD}_{\mathrm{S}_{100 \mathrm{~A}}}$ with CVF shown by the scattered maps. Y-axis is $\mathrm{CVF}$ of primary IBC, and $\mathrm{X}$-axis is the values of both elasticity index and $\mathrm{AOD}_{\mathrm{S}_{100 \mathrm{~A}} \mathrm{i}}$ in primary IBC. The graph shows that both the elasticity and the S100A4 expression are positively correlated with collagen deposition, which means that collagen deposition was higher in stiff masses with higher elasticity parameters, as well as in those masses with higher S100A4 expression. Emax, the maximum stiff value; Emean, the mean stiff value; EmeanR, the ratio of Emean between mass and parenchyma; $\mathrm{AOD}_{\mathrm{S}_{100 \mathrm{~A}} \text {, the average }}$ optical density of S100A4; CVF, collagen volume fraction; IBC, invasive breast carcinoma.

were observed between the two groups concerning tumor type, intrinsic subtype, or expression of estrogen receptor (ER), progesterone receptor (PR), human epidermal growth factor receptor 2 (HER2), p53, CK5/6, and E-cadherin, or for the biological markers CA153 and CEA.

\section{US and SWE features}

Comparisons of US features and SWE elasticity indexes of IBC between the LNM+ and LNM- groups are shown in Table 2. For features of conventional US, masses in the $\mathrm{LNM}+$ group were characterized by irregular shape $(\mathrm{P}=0.001)$. Most IBCs in the LNM+ group presented shadowing or no posterior features, while the posterior features in the LNM- the group were variable $(\mathrm{P}=0.033)$. There were no differences in orientation, margin, calcification, or vascularity features between the two groups. For SWE, elasticity indexes, including Emax, Emean, and EmeanR, were all significantly higher in the $\mathrm{LNM}+$ group than in the LNM- group $(\mathrm{P}<0.01)$.
The best cutoff values of SWE for diagnosing lymphatic metastasis were as follows: $111.05 \mathrm{kPa}$ for Emax, $79.80 \mathrm{kPa}$ for Emean, and 6.89 for EmeanR. The ROC curve is shown in Figure 4. The area under the curve (AUC), sensitivity (Sen), specificity (Spe), positive predictive value (PPV), negative predictive value (NPV), and diagnostic accuracy (ACC) with optimal cutoff values are shown in Table 3.

\section{Discussion}

Considering that preoperative knowledge of axillary lymph node status is crucial for both the use of neoadjuvant chemotherapy and appropriate surgical management, there is a need for the development of imaging tools specifically for LNM diagnosis (9). While there has always been a debate about the need for clearance of all positive axillary lymph nodes, efforts to preoperatively diagnose every malignant lymph node are being discouraged by some surgeons (10). As such, more reliable indicators for preoperative positive lymph node assessment are needed. One study has shown lesion stiffness to be a predictor of cervical lymphatic metastasis in papillary thyroid carcinoma (PTC), and reported that the optimal cutoff values of Emax and Emean for the prediction of central LNM were 138 $\mathrm{kPa}$ and $124 \mathrm{kPa}$ respectively, and the optimal cutoff values of Emin for the prediction of lateral LNM was $63 \mathrm{kPa}$ (11). Evans et al. reported that mean stiffness at SWE is an independent predictor of axillary LNM in women with IBC (3). Although they did not show the optimal cutoff value of SWE, they described in detail the mean of mean stiffness values of IBC with 3 nodal statuses: $114 \mathrm{kPa}$ for the negative node group, $141 \mathrm{kPa}$ for the $1-3$ nodes positive group, and $156 \mathrm{kPa}$ for the 4 or more nodes positive group. Compared with the mean stiffness values, whether IBC with positive nodes or IBC with negative nodes, the corresponding Emean values in our study were lower $(101.16 \pm 38.91 \mathrm{kPa}$ for $\mathrm{LNM}+$ and $65.41 \pm 25.26 \mathrm{kPa}$ for LNM-). The difference may result from the following 3 factors: the included patients (who underwent neoadjuvant chemotherapy before surgery were excluded in our study); the setting of the instrument, including the probe, the display scale, ROI, and Q-Box (information for which was absent in Evan's report); and the operator-dependent variations. Furthermore, convincing pathological and molecular data supporting these indicators are needed.

Collagen remodeling resulting from the invasive behavior of tumor cells into the stroma periphery cause normally flexible fiber to become stiff, which is thought 
Table 1 Comparisons of clinicopathological characteristics in IBCs between the LNM- and LNM+ groups

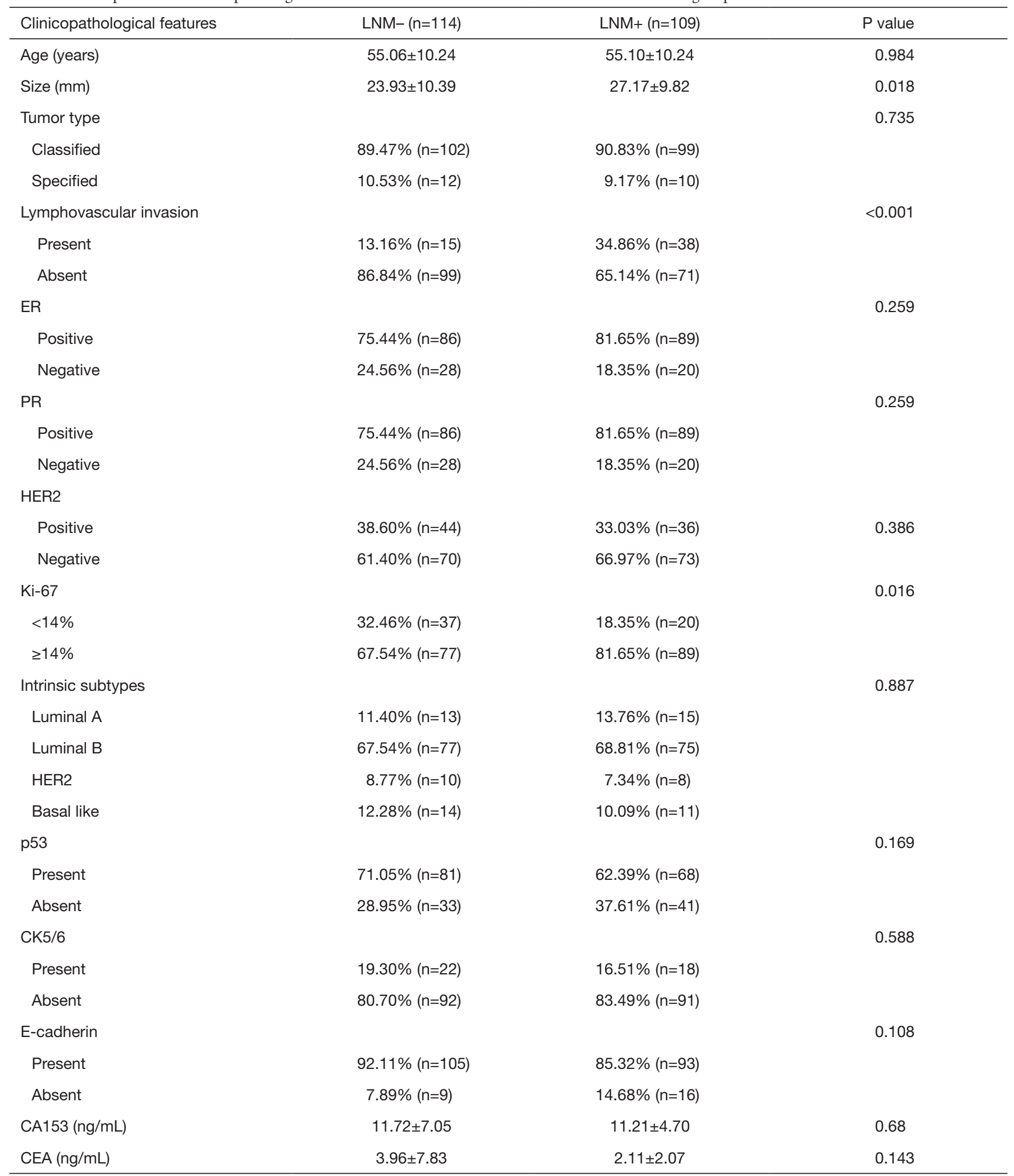

IBC, invasive breast cancer; LNM, axillary lymph node metastasis; ER, estrogen receptor; PR, progesterone receptor; HER2, human epidermal growth factor receptor 2 . 
Table 2 Comparisons of US features and SWE values in IBCs between the LNM- and LNM+ groups

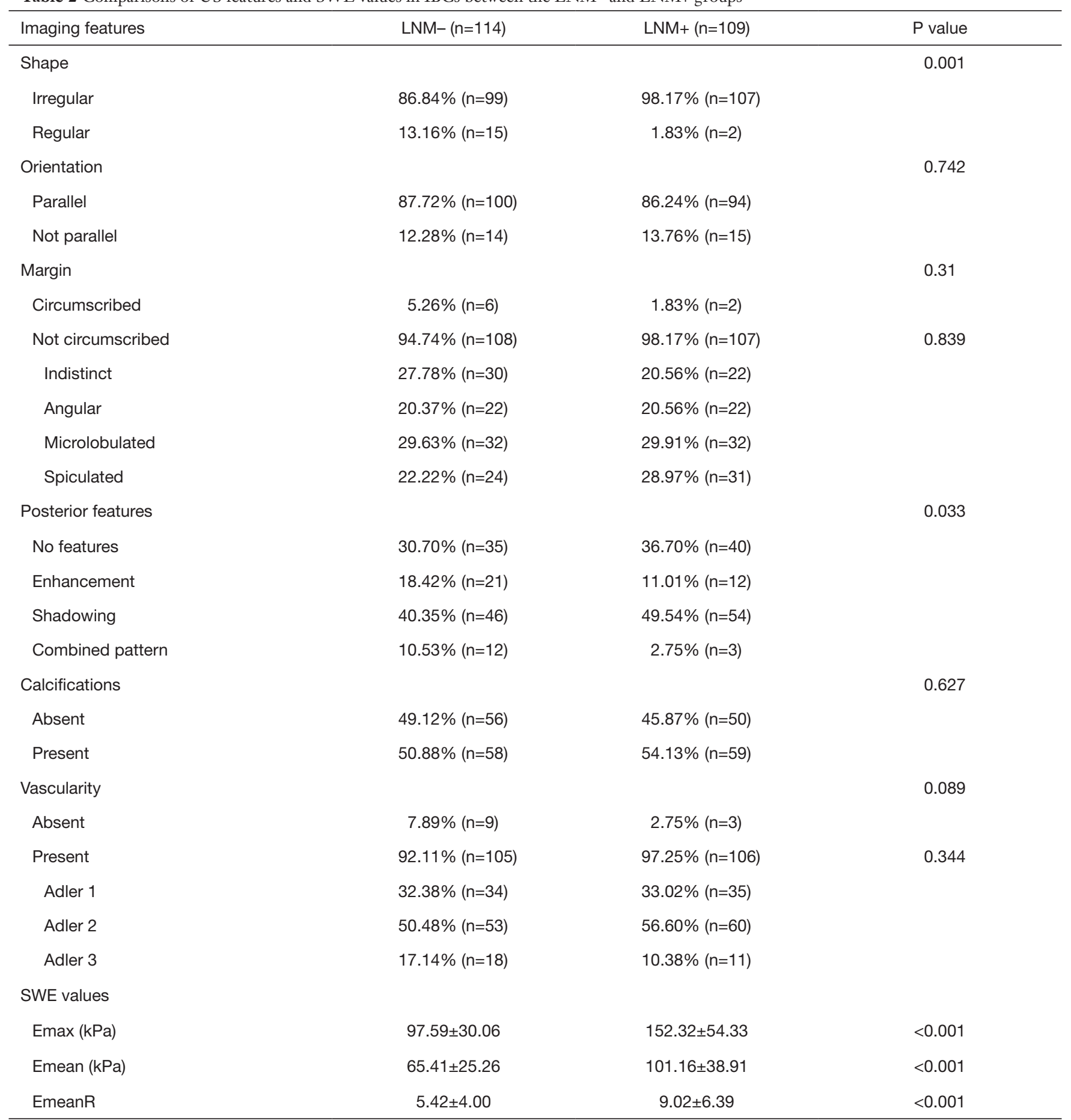

IBC, invasive breast cancer; LNM, axillary lymph node metastasis; US, ultrasound; SWE, shear wave elastography.

to be the chief pathological basis for SWE, and this differentiates malignant lesions from the benign ones (12). We correlated SWE indexes with collagen deposition in
126 primary IBC tissue samples, and all three parameters were positively correlated with CVF, with Emax showing excellent reliability, followed by Emean and EmeanR. The 
IBC masses are mostly heterogeneous and the normal breast parenchyma is varied, so Emean and EmeanR are secondary indicators compared to Emax. Also, the mean CVF of 65 tissue samples from primary IBCs that were LNM+ was significantly higher than that in the 61 LNMsamples, indicating a greater extent of collagen remodeling and greater cancer-associated collagen composition in the LNM+ group. Obviously, the discrepancy in collagen deposition of primary IBC between the LNM+ and LNM- groups was quantitatively well presented by SWE. Therefore, we initially investigated if there are molecules playing a key role in regulating cancer-associated collagen deposition that simultaneously promotes metastasis.

S100A4, encoded by S100A4 and located on a frequently rearranged gene cluster on chromosome 1q21 in humans, is best known as a metastasis-associated protein in malignant

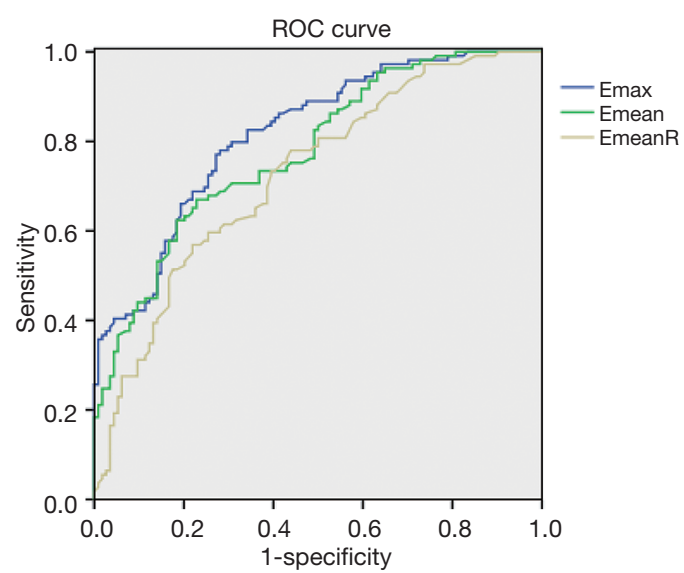

Figure 4 The ROC curve of SWE for diagnosing IBC lymphatic metastasis. The ROC curve shows that Emax exhibited the best diagnostic performance with the largest AUC, followed by Emean with the second-largest AUC and EmeanR with the smallest AUC. ROC, receiver operating characteristic; SWE, shear wave elastography; IBC, invasive breast carcinoma; AUC, the area under the curve. tumors (13). The expression of S100A4 is positively correlated with cancer progression and metastasis in many cancer tissues, including breast, prostate, ovarian, and other cancers (14-18). Therefore, we searched GEO datasets to examine whether there is a difference in S100A4 expression between primary IBCs that are $\mathrm{LNM}+$ and those that are LNM-. As expected, S100A4 expression was significantly higher in the LNM+ group. Next, 65 IBC tissue samples that were $\mathrm{LNM}+$ and $61 \mathrm{IBC}$ tissue samples that were LNM- were validated for immunohistochemical analysis; the mean $\mathrm{AOD}_{\mathrm{S}_{100 \mathrm{~A}}}$ of samples in the $\mathrm{LNM}+$ group was significantly higher than that in the LNM- group. Also, we examined the correlation between $\mathrm{AOD}_{\mathrm{S}_{100 \mathrm{~A}} 4}$ and $\mathrm{CVF}$ in the tissue samples, and the results revealed an excellent reliable correlation between these parameters, indicating that S100A4 may represent a positive regulatory factor promoting cancer-associated collagen deposition. Studies have reported that $\mathrm{S} 100 \mathrm{~A} 4$ plays a vital role in various nontumor diseases, such as fibrosis, inflammation, immune response, and neuroprotection, where it regulates tissue fibrosis via different signaling pathways and stimulates fibroblasts to secrete collagen, inducing collagen deposition (19-24). These results suggest that S100A4 may regulate collagen deposition in tumor disorders via other signaling pathways; we have begun to investigate the possible signaling mechanisms using in vivo and in vitro methods.

Further, 223 patients with IBC were prospectively observed and were divided into the LNM+ group containing 109 women and the LNM- group containing 114 women. SWE elasticity indexes, including Emax, Emean, and EmeanR of primary IBC lesions, were compared, presenting significantly higher values in the $\mathrm{LNM}+$ group than in the LNM- group. The best cutoff values of the SWE parameters for diagnosing lymphatic metastasis were calculated as follows: $111.05 \mathrm{kPa}$ for Emax, $79.80 \mathrm{kPa}$ for Emean, and 6.89 for EmeanR. According to ROC analysis, Emax exhibited the best diagnostic performance with an AUC $[95 \%$ confidence interval (CI)] of $0.82(0.76-0.87)$,

Table 3 The diagnostic performance of SWE for predicting axillary lymphatic metastasis with the best cutoff values

\begin{tabular}{lcccccccc}
\hline SWE & Cutoff value & AUC $(95 \% \mathrm{Cl})$ & P value & Sen & Spe & ACC, \% & PPV, \% & NPV, \% \\
\hline Emax & $111.05 \mathrm{kPa}$ & $0.82(0.76-0.87)$ & $<0.001$ & 0.78 & 0.72 & 74.88 & 73.04 & 76.85 \\
Emean & $79.80 \mathrm{kPa}$ & $0.78(0.72-0.84)$ & $<0.001$ & 0.67 & 0.77 & 72.20 & 73.74 & 70.97 \\
EmeanR & 6.89 & $0.73(0.66-0.79)$ & $<0.001$ & 0.57 & 0.78 & 67.71 & 71.26 & 65.44 \\
\hline
\end{tabular}

SWE, shear wave elastography; AUC, area under the curve; Cl, confidence interval; Sen, sensitivity; Spe, specificity; ACC, accuracy; PPV, positive predictive value; NPV, negative predictive value. 
following by an Emean of 0.78 (0.72-0.84) and an EmeanR of $0.73(0.66-0.79)$. In addition to the shape and posterior features on the US, the SWE elasticity indexes could supply nodal status, a powerful imaging method, although the ACC, as well as Sen and Spe, were lower than those previously reported for benign and malignant breast tumor differentiation (25). As a reference, the US of the axilla with the biopsy of abnormal lymph nodes is diagnostic for no more than $50 \%$ of positive node patients (26). For clinicopathological characteristics in this study, most of the features exhibited no difference between the two groups except for tumor size, lymphovascular invasion, and Ki67 expression, and these 3 variables are well-established as unfavorable prognostic factors, consequently presenting in higher levels in the LNM+ group.

This study has some limitations. When analyzing the pathology basis and possible key molecules, we excluded samples for undergoing neoadjuvant chemotherapy before surgery, which might have introduced bias into case selection. The second limitation is that the samples in this study used for determining diagnostic cutoff values of SWE were relatively small in number, and additional data should be included in future studies. Furthermore, the mechanism of the "SWE-collagen-S100A4-LNM" chain needs further validation in molecular biological experiments. An important final limitation is linked to the operatordependent variations related to the use of SWE.

In conclusion, high SWE elasticity indexes, especially for Emax and Emean, could indicate axillary LNM of IBCs. Collagen deposition should be the pathological basis for SWE diagnosis, and S100A4 may be a regulatory molecule that simultaneously promotes metastasis and increases the stiffness of primary tumors.

\section{Acknowledgments}

Funding: This study was supported by grants from the National Natural Science Foundation of China (No. 81630048) and the Fundamental Research Fund for the Provincial Universities (No. 31041180108).

\section{Footnote}

Conflicts of Interest: The authors have no conflicts of interest to declare.

Ethical Statement: The present study was approved by the Ethics Committee of the Harbin Medical University Cancer
Hospital (No. KY2016-34) and was conducted following the ethical standards of the institutional and national research committee. Informed consent was waived owing to the retrospective nature of the study.

Open Access Statement: This is an Open Access article distributed in accordance with the Creative Commons Attribution-NonCommercial-NoDerivs 4.0 International License (CC BY-NC-ND 4.0), which permits the noncommercial replication and distribution of the article with the strict proviso that no changes or edits are made and the original work is properly cited (including links to both the formal publication through the relevant DOI and the license). See: https://creativecommons.org/licenses/by-nc-nd/4.0/.

\section{References}

1. Suvannarerg V, Chitchumnong P, Apiwat W, Lertdamrongdej L, Tretipwanit $M$, Pisarnturakit P, Sitthinamsuwan P, Thiravit S, Muangsomboon K, Korpraphong P. Diagnostic performance of qualitative and quantitative shear wave elastography in differentiating malignant from benign breast masses, and association with the histological prognostic factors. Quant Imaging Med Surg 2019;9:386-98.

2. Seewaldt V. ECM stiffness paves the way for tumor cells. Nat Med 2014;20:332-3.

3. Evans A, Ranchhaus P, Whelehan P, Thomson K, Purdie CA, Jordan LB, Michie CO, Thompson A, Vinnicombe S. Does shear wave ultrasound independently predict axillary lymph node metastasis in women with invasive breast cancer? Breast Cancer Res Treat 2014;143:153-7.

4. Prasmickaite L, Tenstad EM, Pettersen S, Jabeen S, Egeland EV, Nord S, Pandya A, Haugen MH, Kristensen VN, Børresen-Dale AL, Oslo Breast Cancer Research Consortium (OSBREAC), Engebråten O, Mælandsmo GM. Basal-like breast cancer engages tumor-supportive macrophages via secreted factors induced by extracellular S100A4. Mol Oncol 2018;12:1540-58.

5. Klingelhöfer J, Senolt L, Baslund B, Nielsen GH, Skibshøj I, Pavelka K, Neidhart M, Gay S, Ambartsumian N, Hansen BS, Petersen J, Lukanidin E, Grigorian M. Up-regulation of metastasis-promoting S100A4 (Mts1) in rheumatoid arthritis: putative involvement in the pathogenesis of rheumatoid arthritis. Arthritis Rheum 2007;56:779-89.

6. Tomcik M, Palumbo-Zerr K, Zerr P, Avouac J, Dees C, Sumova B, Distler A, Beyer C, Cerezo LA, Becvar R, 
Distler O, Grigorian M, Schett G, Senolt L, Distler JHW. S100A4 amplifies TGF- $\beta$-induced fibroblast activation in systemic sclerosis. Ann Rheum Dis 2015;74:1748-55.

7. D'Orsi CJ, Sickles EA, Mendelson EB, Morris EA. ACR BI-RADS atlas, breast imaging reporting and data system. Reston: American College of Radiology, 2013.

8. Anvari A, Halpern EF, Samir AE. Essentials of statistical methods for assessing reliability and agreement in quantitative imaging. Acad Radiol 2018;25:391-6.

9. Wang NN, Yang ZJ, Wang X, Chen LX, Zhao HM, Cao WF, Zhang B. A mathematical prediction model incorporating molecular subtype for risk of non-sentinel lymph node metastasis in sentinel lymph node-positive breast cancer patients: a retrospective analysis and nomogram development. Breast Cancer 2018;25:629-38.

10. Gabriel E, Attwood K, Young J, Cappuccino H, Kumar S. Impact of American College of Surgeons Oncology Group Z11 on surgical training at an academic cancer center. J Surg Res 2016;201:266-71.

11. Park AY, Kim JA, Son EJ, Youk JH. Shear-wave elastography for papillary thyroid carcinoma can improve prediction of cervical lymph node metastasis. Ann Surg Oncol 2016;23:722-9.

12. Acerbi I, Cassereau L, Dean I, et al. Human breast cancer invasion and aggression correlates with ECM stiffening and immune cell infiltration. Integr Biol (Camb) 2015;7:1120-34.

13. Fei F, Qu J, Li CY, Wang XL, Li YW, Zhang SW. Role of metastasis-induced protein S100A4 in human non-tumor pathophysiologies. Cell Biosci 2017;7:64-74.

14. Qu S, Wu JH, Bao QY, Yao B, Duan R, Chen X, Li LY, Yuan $\mathrm{HY}$, Jin YC, Ma CY. Osterix promotes the migration and angiogenesis of breast cancer by upregulation of S100A4 expression. J Cell Mol Med 2019;23:1116-27.

15. Ruma IMW, Kinoshita R, Tomonobu N, Inoue Y, Kondo E, Yamauchi A, Sato H, Sumardika IW, Chen Y, Yamamoto KI, Murata H, Toyooka S, Nishibori M, Sakaguchi M. Embigin promotes prostate cancer progression by S100A4dependent and-independent mechanisms. Cancers (Basel) 2018;10:239-63.

16. Link T, Kuhlmann JD, Kobelt D, Herrmann P, Vassileva YD, Kramer M, Frank K, Göckenjan M, Wimberger P, Stein U. Clinical relevance of circulating MACC1 and S100A4 transcripts for ovarian cancer. Mol Oncol 2019;13:1268-79.

17. Smith BN, Bhowmick NA. Role of EMT in metastasis and therapy resistance. J Clin Med 2016. doi: 10.3390/ jcm5020017.
18. Ishikawa $M$, Osaki $M$, Yamagishi $M$, Onuma K, Ito H, Okada F, Endo H. Correlation of two distinct metastasisassociated proteins, MTA1 and S100A4, in angiogenesis for promoting tumor growth. Oncogene 2019;38:4715-28.

19. Li Y1, Bao J, Bian Y, Erben U, Wang P, Song K, Liu S, Li Z, Gao Z, Qin Z. S100A4+ macrophages are necessary for pulmonary fibrosis by activating lung fibroblasts. Front Immunol 2018;9:1776.

20. Xia H, Gilbertsen A, Herrera J, Racila E, Smith K, Peterson M, Griffin T, Benyumov A, Yang LB, Bitterman $\mathrm{PB}$, Henke CA. Calcium-binding protein S100A4 confers mesenchymal progenitor cell fibrogenicity in idiopathic pulmonary fibrosis. J Clin Invest 2017;127:2586-97.

21. Zhang J, Hou S, Gu J, Tian T, Yuan Q, Jia J, Qin Z, Chen Z. S100A4 promotes colon inflammation and colitis-associated colon tumorigenesis. Oncoimmunology 2018;7:e1461301.

22. Zhang YH, Ma DQ, Ding DP, Li J, Chen LL, Ao KJ, TianYY. S100A4 gene is crucial for methionine-cholinedeficient diet-induced non-alcoholic fatty liver disease in mice. Yonsei Med J 2018;59:1064-71.

23. Braumann S, Thottakara T, Stücker S, ReischmannDüsener S, Krämer Elisabeth, Groß J, Hirt MN, Doroudgar S, Carrier L, Friedrich FW. S100A4 as a target of the E3-ligase Asb2 $\beta$ and its effect on engineered heart tissue. Front Physiol 2018;9:1292-303.

24. Pankratova S, Klingelhofer J, Dmytriyeva O, Owczarek S, Renziehausen A, Syed N, Porter AE, Dexter DT, Kiryushko D. The S100A4 protein signals through the ErbB4 receptor to promote neuronal survival. Theranostics 2018;8:3977-90.

25. Huang R, Jiang L, Xu Y, Gong Y, Ran H, Wang Z, Sun Y. Comparative diagnostic accuracy of contrast-enhanced ultrasound and shear wave elastography in differentiating benign and malignant lesions: a network meta-analysis. Front Oncol 2019;9:102.

26. Alvarez S, Añorbe E, Alcorta P, López F, Alonso I, Cortés J. Role of sonography in the diagnosis of axillary lymph node metastases in breast cancer: a systematic review. AJR Am J Roentgenol 2006;186:1342-8.

Cite this article as: Wen X, Yu X, Tian Y, Liu Z, Cheng W, Li H, Kang J, Wei T, Yuan S, Tian J. Quantitative shear wave elastography in primary invasive breast cancers, based on collagen-S100A4 pathology, indicates axillary lymph node metastasis. Quant Imaging Med Surg 2020;10(3):624-633. doi 10.21037/qims.2020.02.18 